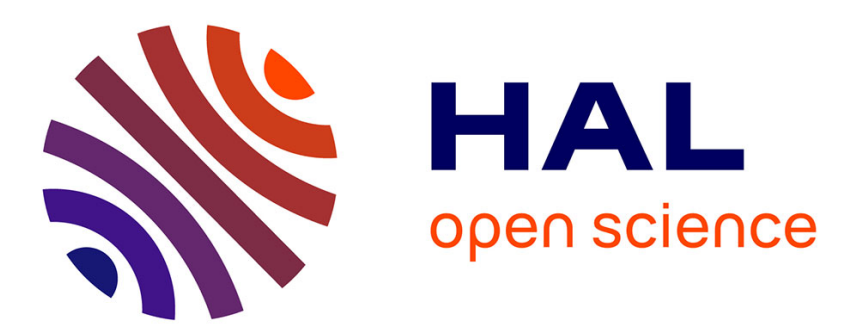

\title{
Role of Self-Organization of Dislocations in the Onset and Kinetics of Macroscopic Plastic Instability
}

Nikolay P Kobelev, Mikhail A Lebyodkin, Tatiana A Lebedkina

\section{To cite this version:}

Nikolay P Kobelev, Mikhail A Lebyodkin, Tatiana A Lebedkina. Role of Self-Organization of Dislocations in the Onset and Kinetics of Macroscopic Plastic Instability. Metallurgical and Materials Transactions A, 2017, 48, pp.965-974. 10.1007/s11661-016-3912-x . hal-03256672

\section{HAL Id: hal-03256672 \\ https://hal.univ-lorraine.fr/hal-03256672}

Submitted on 15 Nov 2021

HAL is a multi-disciplinary open access archive for the deposit and dissemination of scientific research documents, whether they are published or not. The documents may come from teaching and research institutions in France or abroad, or from public or private research centers.
L'archive ouverte pluridisciplinaire HAL, est destinée au dépôt et à la diffusion de documents scientifiques de niveau recherche, publiés ou non, émanant des établissements d'enseignement et de recherche français ou étrangers, des laboratoires publics ou privés. 


\title{
Role of self-organization of dislocations in the onset and kinetics of macroscopic plastic instability
}

\author{
N.P. Kobelev ${ }^{\text {a }}$, M.A. Lebyodkin ${ }^{\text {b,* }}$, T.A. Lebedkina ${ }^{\text {b }}$ \\ ${ }^{\mathrm{a}}$ Institute of Solid State Physics, Russian Academy of Sciences, 142432 Chernogolovka, Russia \\ ${ }^{\mathrm{b}}$ LEM3, UMR CNRS 7239, Université de Lorraine, Ile du Saulcy, 57045 Metz Cedex 01, France
}

\begin{abstract}
The present paper examines two aspects of the problem of critical conditions of jerky flow in alloys, or the Portevin-Le Chatelier (PLC) effect. Recent development of dynamic strain aging (DSA) models proved their capacity to qualitatively reproduce complex non-monotonic behavior of the critical strain, providing that the parameters of theory are allowed to depend on strain. Experimental measurements of such strain dependences have been realized for the first time and used to revise the predictions of the critical strain and stress relaxation kinetics upon abrupt strain-rate changes. On the other hand, it is usually omitted from consideration that the PLC stress serrations can last very short time in comparison with the characteristic time of stress transients. The development of stress drops was studied with the aid of the acoustic emission (AE) technique. It is shown that such macroscopic instabilities are caused by clustering of AE events which otherwise occur all the time, including the periods of smooth plastic flow. The role of synchronization of dislocation avalanches in the development of abrupt stress serrations and its relationship with the predictions of the local DSA models is discussed.
\end{abstract}

* Corresponding author. E-mail address: mikhail.lebedkin@ univ-lorraine.fr

\section{Introduction}

The interaction of dislocations with solute atoms in dilute alloys can lead to instability of uniform plastic flow. This phenomenon, known as the Portevin-Le Chatelier effect (PLC) [1], is manifested by jerky deformation curves and strain localization in deformation bands [2,3]. The generally accepted mechanism of the PLC effect is based on the concept of dynamic strain aging (DSA) considering solute pinning of mobile dislocations arrested on obstacles during the waiting time $t_{w}$ for thermal activation [4-15]. Due to such additional pinning, the strain-rate sensitivity (SRS) of the flow stress $\sigma, S_{f}=\left(\partial \sigma / \partial \ln \left(\dot{\varepsilon}_{p}\right)\right)_{\varepsilon_{p}, T}$, becomes negative in a certain range of strain $\varepsilon_{p}$, strain rate, and temperature $T$, thus giving rise to instability [5].

One of the long-standing challenges concerns an adequate description of the critical conditions for onset of plastic instability, in particular, the critical strain $\varepsilon_{c}$. Early models based on the DSA predicted the so-called "normal" behavior consisting of an increase in $\varepsilon_{c}$ with an increase 
in the applied strain rate $\dot{\varepsilon}_{a}$ or a decrease in temperature [6-8]. Experimental studies showed a non-monotonic dependence including "inverse" behavior at low $\dot{\varepsilon}_{a}$-values (e.g., $\left.[9,16,17]\right)$. Although the latter can be caused by specific mechanisms, e.g., associated with precipitates [18], non-monotonic behavior was recently explained in a unique framework of DSA modified to allow for its dependence on strain $[19,20]$. In spite of this success, several fundamental issues were not fully addressed. It was shown quite early that the negative value of SRS does not suffice to generate a macroscopic instability because a finite time is needed for the strain rate to localize into a deformation band [6]. In [6], the conditions of the growth of a local perturbation of the uniform strain state were examined to find criteria for the onset of flow localization. An important feature of the model is a characteristic relaxation time $\tau$ for the SRS, which is of the order of $t_{w}$ and can be estimated from the transient kinetics of the flow stress upon abrupt changes in $\dot{\varepsilon}_{a}$. The linear stability analysis provided two new criteria corresponding to either oscillating or exponential growth of the small perturbation, respectively, at $\varepsilon_{\phi}$ and $\varepsilon_{\theta}$, where $\varepsilon_{\theta}>\varepsilon_{\phi}>\varepsilon_{0}$, and $\varepsilon_{0}$ is the critical strain obtained for the condition $S_{f}<0$. The available experimental data for $\varepsilon_{c}$ were found to lie between these two criteria. Recent finite-element modeling rendered $\varepsilon_{c}$ values rather close to $\varepsilon_{\theta}$ [20]. In any case, the physical reasons for both the choice between the criteria and the intermediate position of experimental data remained unclear.

Perhaps, there is even less clarity concerning the development of instability which cannot be handled by the linear stability analysis. The numerical simulation of heterogeneous flow implemented in [6] predicted smooth oscillatory instability growth governed by the stress relaxation kinetics. This prediction was corroborated by experimental data for some alloys containing precipitates [21, 22]. However, most experimental data testify to very abrupt stress drops, at least at low enough strain rates [23-27]. The jerky character of stress drops, which gave the name of "jerky flow" to the PLC effect, was also predicted in numerous numerical models [27-33], but the problems of the critical strain and transient stress kinetics mostly remained beyond the scope of these papers. Another phenomenon which is directly related to this problem concerns small stress drops often observed before the onset of the PLC effect and also between large serrations [34-36]. This feature is usually explained in terms of sporadic fluctuations which may eventually trigger macroscopic instabilities [34]. In particular, the temperature and strain-rate domain of the PLC effect were found to be considerably larger than that predicted by the linear stability analysis. However, such small stress drops are much bigger than the stress fluctuations observable on smooth deformation curves of pure materials (e.g., [37]). Simple estimates show that their occurrence requires motion of tens of thousands of dislocations. It is thus clear that the understanding of the onset of the PLC effect requires consideration of conditions for a collective motion of dislocations in ageing alloys.

To be precise, the necessity of taking into account self-organization of dislocations to describe $\mathcal{E}_{c}$ was mentioned very early [11]. However, this issue has not received further consideration. The question of the role of spatial heterogeneity on $\varepsilon_{c}$ predictions was renewed recently [36, 38, 39] due to investigation of acoustic emission (AE) accompanying the PLC effect in an AlMg alloy. As follows from the entirety of investigations of AE during deformation of various materials, including pure crystals (e.g., [37]), an essential part of dislocation motions in solids proceed by avalanches leading to a burst-like character of the AE. The data $[36,38,39]$ testified that the abrupt stress serrations observed in the conditions of the PLC effect at low enough strain rate are associated with clustering of AE bursts which was supposed to reflect synchronization of dislocation avalanches.

The aim of the present study was to revise the problem of the critical strain in the spirit of these findings. For this purpose, a thorough experimental investigation of the SRS and kinetics of 
stress transients as functions of strain was combined with an AE study. ${ }^{1}$ Besides the possibility of detection of individual deformation events unresolved on the deformation curves, the $\mathrm{AE}$ technique provides a high temporal resolution and allows for a high-accuracy estimation of the duration of plastic strain-rate jumps within deformation bands. An accent was put on the interval of low and intermediate strain rates, as it corresponds to both the inverse behavior of $\varepsilon_{c}$ and the observations of the phenomenon of dislocations synchronization during the PLC effect. Besides, very few data on $S_{f}$ and, especially, $\tau$ have been available for this strain-rate range so far.

\section{Experimental}

Tensile samples with an approximately $10 \mathrm{~mm}^{2}$ cross-section and a gauge length in the range from $30 \mathrm{~mm}$ to $50 \mathrm{~mm}$ were prepared from an Al-3\% Mg alloy with a grain size about $30 \mu \mathrm{m}$. All specimens were annealed for $2 \mathrm{~h}$ at $400^{\circ} \mathrm{C}$ and quenched into water. Deformation was carried out in a hard testing machine. A control test with a steel plate $7 \times 7 \mathrm{~cm}^{2}$ in cross-section as a specimen provided an estimate close to $2 \times 10^{7} \mathrm{~N} / \mathrm{m}$ for the machine spring constant in the tensile setup. This value agrees with the combined stiffness $M$ of the "machine-specimen" system, measured as the slope of the elastic part of the stress-strain curve, which rendered values between 20 to $30 \mathrm{GPa}$, depending on the specimen length. The total strain $\varepsilon$ was measured using a contact extensometer with the initial gap from $25 \mathrm{~mm}$ to $45 \mathrm{~mm}$, covering 80 to 90 percent of the specimen gauge length. The plastic strain was calculated using the relationship $\varepsilon_{p}=\varepsilon-\sigma / E$, where $E$ is the Young's modulus of the AlMg alloy.

The procedure of the SRS measurement was similar to that described in [21, 22]. It consists in a sudden change in the mobile grip velocity and recording the transient process. When the new stationary regime is reached, the reverse switching is performed and the transient is recorded again. An advantage of $S_{f}$ determination by the rate switching method is the possibility of collecting data on the same specimen, whereas the approach based on collecting a family of deformation curves for different $\dot{\varepsilon}_{a}$ may be affected by the microstructure variations between different samples. In contrast to the common practice of changing $\dot{\varepsilon}_{a}$ by at least one order of magnitude [21, 22, 40-42], the change by a factor of 3 was applied in the present work, in order to reduce the possible corruption of results because of a strain-rate effect on the microstructure evolution. Such a smaller jump was found to suffice for $S_{f}$ measurements, due to a high accuracy of this method. The switching was organized so that at least two thirds of the total strain was accumulated during deformation at the strain rate chosen as a reference. In most tests one measurement cycle corresponded to a downward-upward sequence of changes in $\dot{\varepsilon}_{a}$, relative to the reference rate. Switching upward-downward was also tried in several tests. Besides, two experiments comprised two-step downward jumps followed by two-step returns.

The experiments aimed at determining $S_{f}$ were carried out in a range $\dot{\varepsilon}_{a}=3 \times 10^{-6} \mathrm{~s}^{-1}-6 \times$ $10^{-5} \mathrm{~s}^{-1}$, corresponding to inverse behavior of $\varepsilon_{c}$ for the Al-3\% Mg alloy (cf. [16,17,36]) and characterized by high enough $\varepsilon_{c}$-values, so that the measurements could be done during macroscopically smooth flow before the onset of PLC serrations. The measurements of $\varepsilon_{c}$ were performed in a wider $\dot{\varepsilon}_{a}$-range, up to $3 \times 10^{-4} \mathrm{~s}^{-1}$. In most of long-lasting tests at a low strain rate the acquisition time was chosen to be equal to $0.5 \mathrm{~s}$. In several experiments a shorter acquisition time of $20 \mathrm{~ms}$ was used to estimate the duration of stress drops in the investigated alloy.

The exact measurement of the stress drop duration was realized using the AE technique. The AE was recorded with a piezoelectric transducer operating in the frequency band $200-900 \mathrm{kHz}$, which was clamped to the greased specimen surface just above the gauge length. The procedure of real-time recording of AE events was described elsewhere (e.g., [36]), as briefly outlined below. An

\footnotetext{
${ }^{1}$ Preliminary results of this study were presented in proceedings [26].
} 
acoustic event is considered to start when the signal exceeds a preset voltage threshold and end when it drops and remains below the threshold longer than a hit definition time (HDT). A hit lockout time (HLT), or dead time, is waited before resuming the measurement, in order to filter out sound reflections. The voltage threshold was chosen at the level of $27 \mathrm{~dB}$ to avoid background noise. The time parameters, HDT $=$ HLT $=300 \mu$ s, were chosen following the recommendations [43] based on the robustness of the events statistics to the variation of criteria used for their individualization.

\section{DSA approach}

\subsection{Basic equations.}

Various models considering $\mathcal{E}_{c}$ are mostly based on the approach $[6,22]$ which advanced the suggestion that the concentration $C_{S}$ of solute atoms on a dislocation cannot instantaneously change to a new steady value upon a change in the local strain rate ("retardation" effect). The plastic strain rate can be written in the form of the Arrhenius equation [6, 22]:

$$
\dot{\varepsilon}_{p}=\dot{\varepsilon}_{0} \exp \left(-H_{c}^{\prime} C_{S}\right) \cdot \exp \left[\left(\sigma-\sigma_{f}\right) / S_{i}\right]
$$

where $H_{c}^{\prime}=\left(\partial H / \partial C_{S}\right) / k T$ [22], $H$ is the enthalpy of activation of dislocation motion, $\sigma_{f}$ denotes the internal stress, and $S_{i}=\left[\partial \sigma / \partial \ln \left(\dot{\varepsilon}_{p}\right)\right]_{\varepsilon_{p}, C_{S}}$ is the instantaneous SRS value. It should be specified that both SRS values have units of stress (see, e.g., [7]). $C_{S}$ evolves to a saturation value $C_{m}$ with the aging time $t_{a}[22]$ :

$$
C_{S}=C_{m}\left[1-\exp \left(-C_{0}\left(t_{a} / t_{0}\right)^{1 / 3} / C_{m}\right]\right. \text {. }
$$

Here, $C_{0}$ is the solute concentration in the alloy and $t_{0}$ is a constant depending on the solute diffusion coefficient. It is suggested that after abrupt changes in strain rate or stress the aging time follows the relaxation law $[6,22]$

$$
d t_{a} / d t=-\left(t_{a}-t_{w}\right) / \tau
$$

where $t_{w}=\Omega / \dot{\varepsilon}_{p}$ is the average waiting time for thermal activation of dislocations, $\Omega$ is the elementary strain introduced in Ref. [8], $\Omega=\rho_{m} b L$, with $b, \rho_{m}$, and $L$ designating the Burgers vector, the mobile dislocations density, and the average distance between obstacles, respectively. The relaxation time $\tau$ is considered to be equal to $t_{w}$. Equation (1) leads to the following expression for the flow stress:

$$
\sigma=\sigma_{f}+S_{i}\left[\ln \left(\dot{\varepsilon}_{p} / \dot{\varepsilon}_{0}\right)+H_{c}^{\prime} C_{S}\right] .
$$

It is usually accepted for the sake of simplicity that

$$
\sigma_{f}=\sigma_{0}+h \varepsilon_{p}
$$

where $\sigma_{0}$ is a constant and $h$ is the work-hardening rate $\partial \sigma / \partial \varepsilon_{p}$. Contrarily to the usual approximation of constant $h$, the function $h\left(\varepsilon_{p}\right)$ was estimated by numerical derivation of experimental deformation curves $\sigma\left(\varepsilon_{p}\right)$. Finally, the "machine" equation reads

$$
\dot{\sigma} / M+\dot{\varepsilon}_{p}=V / l
$$

with $V$ and $l$ designating the mobile grip velocity and the specimen length, respectively. The numerical integration of Eqs. (2-6) and the comparison of integration results with the experimental dependence $\sigma(t)$ provide an estimate of the four parameters, $S_{i}, \Omega, P_{1}=S_{i} H_{c}{ }^{\prime} C_{m}$, and $P_{2}=\left(C_{0} / C_{m}\right) / t_{0}^{1 / 3}$, used to calculate $S_{f}$ as a function of plastic strain (see Sec. 4.2 for detail on the numerical integration).

\subsection{Instability criteria.}

The critical strains $\varepsilon_{0}, \varepsilon_{\phi}$, and $\varepsilon_{\theta}$ defined in Sec. 1 correspond to the following respective conditions $[6-8,21,22]$ : 


$$
\begin{aligned}
& S_{f}<0, \\
& S_{f}<-(h-\sigma) \dot{\varepsilon}_{p} \tau,
\end{aligned}
$$

and

$$
S_{f}<-(h-\sigma) \dot{\varepsilon}_{p} \tau-2 \sqrt{(h-\sigma) \dot{\varepsilon}_{p} \tau S_{i}},
$$

Similar to the above, the relaxation time in Eqs. (8) and (9) is usually taken equal to $t_{w}$. Equation (8) can be rewritten in the form

$$
S_{i}-P_{1} P_{2} t_{a}^{1 / 3} \exp \left(-P_{2} t_{a}^{1 / 3}\right) / 3<-(h-\sigma) \Omega
$$

and Eq. (9) in the form

$$
S_{i}-P_{1} P_{2} t_{a}^{1 / 3} \exp \left(-P_{2} t_{a}^{1 / 3}\right) / 3<-(h-\sigma) \Omega-2 \sqrt{(h-\sigma) \Omega S_{i}} .
$$

Equations (1)-(6) qualitatively describe normal behavior of $\varepsilon_{c}$ for any of the three criteria. To capture inverse behavior, it was suggested to allow for strain dependence of some model parameters $[19,20]$. In particular, it was supposed that the factor $H_{c}^{\prime} C_{m}$ in the parameter $P_{1}$ depends on strain because the conditions of unpinning of a dislocation from solutes are affected by neighboring dislocations. In the present work, $P_{1}$ as well other parameters of the theory were determined experimentally so that such dependence was taken into account in a natural way.

\section{Experimental results and data processing}

4.1. Deformation curves and the accompanying $A E$.

Figures 1 presents an example of a $\sigma(t)$ - curve recorded at $\dot{\varepsilon}_{a}=2 \times 10^{-5} \mathrm{~s}^{-1}$. The corresponding time derivative is traced in the inset to emphasize the observed discontinuities. The deep stress drops occurring at $\varepsilon_{c}$ correspond to the so-called type $C$ behavior typical of the PLC effect at low strain rates [2,17]. It is known that each of such serrations is caused by a short-time high-rate deformation within a localized (sometimes several) band (e.g., [44]). Small stress serrations are clearly seen before $\varepsilon_{c}$. In [34], it was suggested that such serrations are due to surface defects because they are observed in unpolished as-rolled samples but can be suppressed by mechanical polishing. Similarly to these results, mechanical polishing of the studied specimens effectively suppressed the small serrations. However, control tests showed that if it is followed by either chemical polishing or annealing at $400{ }^{\circ} \mathrm{C}$, the serrations are restored and sometimes become even more distinct than in the initial samples. This observation contradicts the conjecture of sporadic fluctuations induced by the surface imperfection.

The nature of both small and large stress drops becomes clearer from the analysis of the accompanying AE. Figure 2 compares the derivative $\dot{\sigma}(t)$ with the respective series of amplitudes $A$ and durations $D$ of AE events in a strain interval corresponding to a well-developed PLC effect. The representation using bars for the values of $A$ and $D$ helps establishing a correspondence between the stress drops and the AE events. It can be seen that the latter are generated everywhere and have similar amplitudes so that the instants of plastic instability are undistinguishable from the intervals of smooth plastic flow with regard to the $A$ - range. At the same time, the stress drops are perfectly correlated with bursts in the AE duration which almost reaches $10 \mathrm{~ms}$, as compared to the general range below approximately $0.3 \mathrm{~ms}$. As suggested in $[36,38]$, this can mean that a macroscopic stress drop is not caused by a single catastrophic dislocation avalanche but occurs through chaining (synchronization) of dislocation avalanches similar to those accompanying the smooth flow. Figure 3 illustrates that the synchronization operates long before $\varepsilon_{c}$ and is very strong during the PLC instability. Indeed, it can be seen in Fig. 3(a) that small stress drops correspond to clustering of AE events. At the same time, there is nothing extraordinary about these hits although several of them show amplitudes and durations somewhat larger than their neighbors. Indeed, similar $A$ and $D$ values are observed during smooth plastic flow, e.g., around 4200 seconds. The accompanying Fig. 3(b) shows that during each large stress drop, the synchronization is so strong 
that one of the hits is extremely long, while no effect on $A$ can be recognized. ${ }^{2}$ It can be supposed that for a given alloy, there is no difference between the elementary dislocation avalanches participating in smooth plastic flow, small stress drops, or large serrations, but the three situations differ as to the degree of correlation between the avalanches.

\subsection{Analysis of the transient kinetics.}

For each specimen, the strain dependences of $S_{i}, \Omega$, and $P_{1}$ were found by numerical integration of Eqs. (2)-(6) over the entire series of transient cycles performed during its deformation. The integration started from a set of $S_{i}, \Omega$, and $P_{1}$ values independently adjusted for each transient cycle and the best fitting functions were found by successive iterations. Figures 4 and 5 show examples of comparison of experimentally observed and calculated transients as functions of plastic strain and time. It can be seen in Fig. 4 that $\tau$ takes on rather large values corresponding to usual estimates of $t_{w}$ for low strain rates. In particular, these data render a value of $\tau=6 \mathrm{~s}$ around $\varepsilon_{p}=0.5 \%$ and $\tau=14$ s close to the onset of the PLC effect, around $\varepsilon_{p}=3 \%$. Both figures demonstrate that the calculated curves practically coincide with the experimental ones, especially for the downward jumps in $\dot{\varepsilon}_{a}$. For upward jumps, the experimental records show somewhat smoother approach to saturation than the theoretical dependences (see Fig. 4). Given its phenomenological nature, Eq. (3) seems to reflect less adequately the real process in this case. Moreover, it is quite natural that the transition from a higher to a lower $\dot{\varepsilon}_{a}$-value and the return transition may have somewhat different kinetics because in the former case, the relaxation process should be mainly controlled by the pinning of dislocations on obstacles, whereas it corresponds to their unpinning in the latter case (cf. [45]). The differences in the kinetics of these two processes were not explored in the present work. The experimental data described below are given for downward transitions. This choice is also due to a more frequent occurrence of sporadic stress drops upon upward transitions, which makes the analysis more difficult. Their higher probability is most likely caused by the rapid growth of the flow stress immediately after the upward $\dot{\varepsilon}_{a}$-jump.

The calculations display good agreement with experiment not only in the case of the time or strain dependences of $\sigma$ but also for the time evolution of $\varepsilon_{p}$ or $\dot{\varepsilon}_{p}$, as illustrated in Fig. 6 . This figure also shows that the evolution of $\dot{\varepsilon}_{p}$ during the transient process is not monotonous.

Immediately after the change in $\dot{\varepsilon}_{a}$, it falls to a value noticeably below the stationary plastic strain rate which is reached afterwards. The same effect occurs upon the return transition: $\dot{\varepsilon}_{p}$ initially increases above the subsequent steady-state value. This effect may also contribute to the increase in the probability of stress drops after the upward change in $\dot{\varepsilon}_{a}$. Therewith, $t_{a}$ evolves monotonously, as illustrated by the time dependence of the ratio $\Omega / t_{a}$ which has the meaning of strain rate ( $\Omega$ can be considered constant on the scale of Fig. 6).

The data on the dependences of $S_{i}, P_{1}$, and $\Omega$ on $\varepsilon_{p}$, where $\varepsilon_{p}$ is taken at the instants of switching the $\dot{\varepsilon}_{a}$, were found to fit unique functions for different samples and strain rates (see, e.g., data for $P_{1}$ in Fig. 7): $S_{i}=S_{0}+S_{1} \varepsilon_{p}^{1 / 2}, \Omega=\Omega_{0}+\Omega_{1} \varepsilon_{p}^{1 / 2}$, and $P_{1}=P_{10}+P_{11} \varepsilon_{p}$, with parameters $S_{0}=(0.275 \pm 0.021) \mathrm{MPa}, S_{1}=(6.07 \pm 0.15) \mathrm{MPa}, P_{10}=(5.6 \pm 0.2) \mathrm{MPa}, P_{11}=(418 \pm 10)$ $\mathrm{MPa}, \Omega_{0}=(4.2 \pm 3.8) \times 10^{-6}$, and $\Omega_{1}=(1.02 \pm 0.03) \times 10^{-3}$. The uncertainties were estimated as the least-square error of calculation of the slopes of the corresponding dependences. It is noteworthy that the results were also virtually independent on whether the measurements were made on the samples demonstrating small stress drops or on the polished samples without small serrations. The

\footnotetext{
${ }^{2}$ A more detailed analysis shows that some superposition amplifying $A$-values can sometimes be detected. Such analysis will be published elsewhere.
} 
estimate of $P_{2}$ approximately equal to $0.55 \mathrm{~s}^{-1 / 3}$ was obtained from comparison of the transient processes for different reference strain rates. Although all these estimates were obtained from measurements in a relatively narrow strain-rate range, their independence of the strain rate is fully consistent with the assumptions of the model [6] and provides a basis for calculation of the dependences of $S_{f}$ on $\varepsilon_{p}$ and $\dot{\varepsilon}_{p}$ and construction of the instability maps in $\left(\varepsilon_{p}, \dot{\varepsilon}_{p}\right)$-coordinates using the criteria (7), (10), and (11).

The results of calculation of $S_{f}\left(\varepsilon_{p}\right)$-dependences for various $\dot{\varepsilon}_{a}$ are presented in Fig. 8. It can be noted that for the investigated $\dot{\varepsilon}_{a}$-range, $S_{f}$ takes on negative values practically from the beginning of plastic flow. Figure 9 compares the results of $\varepsilon_{c}$ measurements with the instability maps. It can be seen that the tested alloy displays "inverse" behavior in the investigated strain-rate range, with $\varepsilon_{c}$ approaching an approximately constant minimum level when the strain rate is increased, similar to earlier studies on various materials. The values of $\varepsilon_{c}$ lie between $\varepsilon_{\phi}$ and $\varepsilon_{\theta}$, in agreement with the data reported in $[6,21,22]$. An additional critical strain designated as $\varepsilon_{S}$ is plotted to indicate the occurrence of the first small stress drops. Such serrations occur very early so that the values of $\varepsilon_{S}$ are rather small and show strong scatter. Globally, the data on $\varepsilon_{S}$ are bounded by $\varepsilon_{0}\left(\dot{\varepsilon}_{p}\right)$-curves corresponding to the simple criterion of negative SRS.

\section{Discussion}

The data presented in Figs. 4-9 testify that the DSA model reproduces rather well the kinetics of plastic flow upon abrupt changes in the imposed strain rate and, therefore, provides adequate estimates for various parameters determining the SRS value and the characteristic relaxation time. In spite of the fine prediction of the kinetics and a good agreement between data for many specimens, as illustrated in Fig. 7, the criteria of plastic instability only qualitatively agree with the experiment. As a whole, the criteria obtained for an oscillatory and exponential growth of a small perturbation allow estimating the lower and the upper limits of $\varepsilon_{c}$, consistent with the totality of results of earlier investigations. As stated in Sec. 1, these two criteria were suggested to consider relaxation kinetics in order to take into account the finite time needed to develop macroscopic flow localizations [6], whereas the negative SRS criterion determines itself the instability of the uniform plastic flow [5]. The following experimental observations and comparison with theory shed light on the physical meaning of these criteria and help understand their limits.

1. In [6], relationships describing the development of plastic instability were derived in the framework proposed to characterize the stress relaxation kinetics in the presence of retardation effects, generally assumed to be dislocation pinning. Using the predictions [6] and the above experimental estimates of the parameters of theory, the estimate of the characteristic time $t_{\varphi}$ of a stress drop can be obtained for strains between the limits determined by Eqs. (10) and (11): $t_{\varphi} \geq t_{\varphi}\left(\varepsilon_{\theta}\right)=\tau \sqrt{\left(S_{f} /[(h-\sigma) \Omega] \times\left(S_{i} / S_{f}\right)\right.}>\tau$. Even if this inequality may not be satisfied for larger strains, $t_{\varphi}$ is close to $\tau$ in this case too, as can be expected for a process governed by the suggested relaxation kinetics. However, it is well known that the duration of sharp stress drops occurring at low and intermediate strain rates is very small. The significance of this discrepancy already follows from mechanical tests despite their low time resolution. Indeed, control tests using short acquisition time to record $\sigma(t)$-curves show that $t_{\varphi}$ is less than $0.1 \mathrm{~s}$ for $\dot{\varepsilon}_{a}$ around $10^{-5} \mathrm{~s}^{-1}$, i.e., at least 2 orders of magnitude smaller than $\tau$ estimated from the tests with $\dot{\varepsilon}_{a}$ jumps. The AE data testify to very fast kinetics of plastic instability corresponding to a millisecond time scale (see Figs. 2 and 3). These data also agree with high-precision measurements with the aid of capacitance strain transducers [23] or infrared thermography [25]. The analysis of the available literature testifies that the conclusion that there exists strong discrepancy between the experimental estimates 
of $\tau$ and $t_{\varphi}$ is valid for many materials almost in the entire strain-rate domain of existence of the PLC effect, perhaps except for the highest strain rates.

2. Assuming that the characteristic relaxation time of deformation processes corresponds to the extremely short duration of the development of plastic instability, criteria (8) and (9) are reduced to the simple criterion (7) of a negative SRS. As follows from Figs. 8 and 9, this condition is fulfilled practically from the beginning of deformation. It is thus natural to suggest that small serrations which occur virtually immediately after the elastoplastic transition are also a manifestation of the PLC effect. Herewith, the large scatter of the data for $\varepsilon_{S}$ may be caused by the influence of the initial microstructure which can strongly vary between samples. This conjecture is corroborated by the control tests which proved that the small serrations suppressed by mechanical polishing reappear after chemical polishing or annealing. It can be supposed that the "smoothing" effect of the mechanical polishing is not due to removal of surface defects, as suggested in [34], but to an increase in the defects density near the surface. Indeed, the latter may homogenize plastic deformation by either hindering the progress of weak deformation bands [46] or, alternatively, providing dislocation sources to relax the local stresses. Finally, the suggestion that small and large serrations are of the same nature also agrees with their similar duration that can thus be supposed to be controlled by the same mechanism of development of plastic instability.

3. The AE measurements provide a direct proof of the above conjecture. Indeed, the data of Fig. 3 confirm that plastic flow is essentially an avalanche-like process and demonstrate that two kinds of serrations only differ by the efficiency of clustering of dislocation avalanches.

It is clear from these observations that at least at low enough strain rates characterized by abrupt stress drops, an adequate description of the PLC instability should combine two mechanisms: (i) the DSA which determines the loss of stability of uniform flow when the SRS becomes negative and (ii) the phenomenon of synchronization of the dislocation dynamics determining the formation of a deformation band during a stress drop. The success of the criteria taking into account the growth of a small perturbation is likely due to the fact that they mimic the effect of synchronization of dislocations. In particular, exponential perturbation growth corresponds to the complete synchronization of dislocations. It is thus not surprising that this criterion somewhat overestimates $\varepsilon_{c}$. Moreover, general models of dynamical systems showed that synchronization phenomena can occur in systems with negative rate sensitivity [47]. It is therefore possible that in the case of plastic flow, the conditions of synchronization may correlate with the instability criteria.

An additional argument in favor of this viewpoint is the absence of continuity between the evolution of small serrations and the sudden occurrence of deep drops. It is difficult to explain on the sole basis of the DSA model the parameters of which continuously change with strain. As can be seen from Fig. 1, the size of small serrations grows initially, and the extrapolation to large strains makes one think that they could have reached the level corresponding to the "classical" PLC instability. However, they stop growing after some deformation and become rarer and less regular before the onset of the PLC effect. Such a discontinuous change of behavior could be explained as stemming from the change of conditions for dislocations synchronization, as suggested in the following scheme.

An individual avalanche of dislocations, such as those generating short $\mathrm{AE}$ events, is not powerful enough to provide a visible drop in the flow stress. The macroscopic plastic instability requires synchronization of dislocation avalanches. Its development is not determined by the relaxation time $\tau$, as is commonly believed, but by the time necessary for the development of the synchronization process, which can be much smaller (cf. [11]). The internal stress is low and rather heterogeneous at the initial stage of deformation. Hence, there is a low probability that the dislocation avalanches generated in the regions of local stress concentrations will trigger other nearby avalanche events. Thus, their synchronization will be weak and occur sporadically, giving rise to small serrations. The resulting work hardening leads to two effects. On the one hand, the increasing number of obstacles to dislocation motion prevents the mobilization of dislocations and 
thus inhibits the process of synchronization. This change is likely to be the reason of the diminishing frequency of small serrations. On the other hand, the same reason leads to both an increase and homogenization of internal stresses. Also, the density of the mobile dislocations grows with deformation. Both these factors can finally create conditions for strong synchronization of dislocations and result in the occurrence of larger stress drops ${ }^{3}$.

The suggested scheme would work at low enough strain rates when relaxation time $\tau$ is greater than the time of synchronization. It is noteworthy that the DSA model might provide a sufficient framework to predict the critical strain if the characteristic synchronization time is greater or comparable with the relaxation time. Then, both the development of strain localization and the return to the steady state will be controlled by the same relaxation time $\tau$. This may be the case of high strain-rate behavior which remained beyond the scope of this paper. Besides, it may concern industrial alloys in which the appearance of the PLC serrations at low strain rates displays a smooth oscillatory character predicted by the DSA model. This difference with regard to binary alloys may be caused by precipitates that impede the correlation of the dislocation dynamics so that the plastic flow localization should be less pronounced. Indeed, recent observations proved that the precipitates promote deformation band propagation in a wide strain-rate range, whereas it is only observed at high strain rates in binary alloys [48]. The local DSA model might remain adequate in a larger strain-rate range for such materials.

\section{Conclusions}

The question of the limits of the DSA models in reproducing the critical strain for the onset of the PLC effect is usually formulated as "Why can't these models do a better job predicting critical strains?" Taking into account that macroscopic stress drops require collective motion of hundreds of thousands of dislocations, it can otherwise be asked why the local models that do not consider self-organization of dislocations and overestimate by orders of magnitude the stress drop duration are at all able to provide satisfactory results. The present paper tried to accommodate these points of view by combining the traditional experimental approach to the verification of the predictions of DSA models with an acoustic emission study of the collective nature of the dislocation dynamics.

In summary, the following conclusions can be drawn:

1. Strain dependences of various parameters of DSA models are experimentally determined for the first time using a typical AlMg alloy. These data allowed for a thorough revision of the DSA model predictions.

2. The results obtained show that such models adequately describe the relaxation kinetics upon step-wise changes of the strain rate. At the same time, the values of the critical strain for the onset of the PLC effect only qualitatively agree with the experimentally measured values.

3. The kinetics of the stress drop can be much faster than the model prediction and, therefore, the conditions of the nucleation of the corresponding deformation band must be controlled by a different mechanism, at least at low enough strain rates.

4. The experimental data, in particular, behavior of the AE accompanying the plastic deformation testify that this mechanism can be the phenomenon of synchronization of deformation processes.

5. The good prediction of the critical strain using the criterion of exponential growth of a small perturbation, as well some systematic overestimation of the predicted values, is likely to be due to the fact that this criterion corresponds to a requirement of the complete synchronization of dislocations.

\footnotetext{
${ }^{3}$ From the first view, the perfect synchronization could even produce a catastrophic process involving the whole sample. However, this process is stopped because of the fall in the applied stress due to the elastic reaction of the deformation machine when the plastic strain rate exceeds the imposed strain rate (see Eq. (6)).
} 


\section{Acknowledgements}

This work was supported by the Region Lorraine (France) and the Center of Excellence "LabEx DAMAS" through the French State program "Investment in the future" (Grant ANR-11LABX-0008-01 of the French National Research Agency).

\section{References}

1. A. Portevin and F. Le Chatelier: C. R. Acad. Sci. Paris, 1923, vol. 176, pp. 507-10.

2. J.M. Robinson: Int Mater. Rev., 1994, vol. 39, pp. 217-27.

3. Y. Yilmaz: Sci. Technol. Adv. Mater., 2011, vol. 12, p. 063001(16).

4. A. Van den Beukel: Phys. Stat. Sol. (a), 1975, vol. 30, pp. 197-206.

5. P. Penning: Acta metall., 1972, vol. 20, pp. 1169-75.

6. P.G. McCormick: Acta Metall., 1988, vol. 36, pp. 3061-67.

7. Y. Estrin and L.P. Kubin: J. Mech. Behavior Mater., 1990, vol. 2, pp. 255-92.

8. L.P. Kubin and Y. Estrin: Acta Metall. Mater., 1990, vol. 38, pp. 697-708.

9. R. Král and P. Lukáč: Mater. Sci. Eng. A, 1997, vol. 234-236, pp. 786-89.

10. G. Horvath, N.G. Chinh, J. Gubicza, and J. Lendvai: Mater. Sci. Eng. A, 2007, vol. 446, pp. 186-92.

11. P. Hähner: Mater. Sci. Eng. A, 1996, vol. 207, pp. 208-15, 216-23.

12. P. Hähner, A. Ziegenbein, E. Rizzi, and H. Neuhäuser: Phys. Rev. B, 2002, vol. 65, pp.

134109(20).

13. J. Schlipf: Scr. Metall. Mater., 1994, vol. 31, pp. 909-14.

14. F. Springer, A. Nortmann, and Ch. Schwink: Phys. Stat. Sol. A, 1998, vol. 170, pp. 63-81.

15. R.C. Picu and D. Zhang: Acta Mater., 2004, vol. 52, pp. 161-71.

16. J. Balík: Mater. Sci. Eng. A, 2001, vol. 316, pp. 102-08.

17. T.A. Lebedkina and M.A. Lebyodkin: Acta Mater., 2008, vol. 56, pp. 5567-74.

18. Y. Brechet and Y. Estrin: Acta Metall. Mater., 1995, vol. 43, pp. 955-63.

19. T. Böhlke, G. Bondár, Y. Estrin, and M.A. Lebyodkin: Comp. Mater. Sci., 2008, vol. 44, 107688.

20. M. Mazière and H. Dierke: Comp. Mat. Sci., 2012, vol. 52, pp. 68-72.

21. C.P. Ling and P.G. McCormick: Acta Metall. Mater., 1990, vol. 38, pp. 2631-35.

22. C.P. Ling and P.G. McCormick: Acta Metall. Mater., 1993, vol. 41, pp. 3127-31.

23. R.B. Schwarz and L.L. Funk: Acta metall., 1985, vol. 33, pp. 295-307.

24. M. A. Lebyodkin and T. A. Lebedkina: Phys. Rev. E, 2006, vol. 73, pp. 036114(8).

25. H. Ait-Amokhtar, C. Fressengeas, and S. Boudrahem: Mater. Sci. and Eng. A, 2008, vol. 488, pp. 540-46.

26. T.A. Lebedkina, N.P. Kobelev, and M.A. Lebyodkin: Mater. Sci. Forum, 2014, vol. 783-786, pp. 198-203.

27. M.A. Lebyodkin, Y. Bréchet, Y. Estrin, and L.P. Kubin: Phys. Rev. Lett., 1995, vol. 74, pp. 4758-61.

28. P.G. McCormick and C.P. Ling: Acta Metall. Mater., 1995, vol. 43, pp. 1969-77.

29. S. Kok, A.J. Beaudoin, D.A. Tortorelli, and M. Lebyodkin: Model Sim. Mat. Sci. Eng., 2002, vol. 10, pp. 745-63.

30. E. Rizzi and P. Hähner: Inter. J. Plast., 2004, vol. 20, pp. 121-65.

31. G. Lasko, P. Hähner, and S. Schmauder: Model Sim. Mater. Sci. Eng., 2005, vol. 13, 645-56.

32. G. Ananthakrishna: Phys. Reports, 2007, vol. 440, pp. 113-239.

33. S. Varadhan, A.J. Beaudoin, and C. Fressengeas: J. Mech. Phys. Solids, 2009, vol. 57, pp. 173348.

34. M. Abbadi, P. Hähner, and A. Zeghloul: Mater. Sci. Eng. A, 2002, vol. 337, pp. 194-201.

35. D. Thevenet, M. Mliha-Touati, and A. Zeghloul: Mater. Sci. Eng. A, 1999, vol. 266, pp. 175-82.

36. M.A. Lebyodkin, N.P. Kobelev, Y. Bougherira, D. Entemeyer, C. Fressengeas, V.S. Gornakov,

T.A. Lebedkina, and I.V. Shashkov: Acta Mater., 2012, vol. 60, pp. 3729-40. 
37. J. Weiss, T. Richeton, F. Louchet, F. Chmelik, P. Dobron, D. Entemeyer, M. Lebyodkin, T. Lebedkina, C. Fressengeas, and R.J. McDonald: Phys. Rev. B, 2007, vol. 76, pp. 224110(8).

38. M.A. Lebyodkin, N.P. Kobelev, Y. Bougherira, D. Entemeyer, C. Fressengeas, T.A. Lebedkina, and I.V. Shashkov: Acta Mater., 2012, vol. 60, pp. 844-50.

39. I.V. Shashkov, M.A. Lebyodkin, and T.A. Lebedkina, Acta Mater., 2012, vol. 60, pp. 6842-50.

40. R.C. Picu, G. Vincze, J.J. Gracio, and F. Barlat: Scripta Mater., 2006, vol. 54, pp. 71-75.

41. F. Ozturk, H. Pekel, and H.S. Halkaci: J. Mater. Eng. Perform., 2011, vol. 20, pp. 77-81.

42. D. Wowk and K. Pilkey: Mater. Sci. Eng. A, 2009, vol. 520, pp. 174-78.

43. M. A. Lebyodkin, I. V. Shashkov, T. A. Lebedkina, K. Mathis, P. Dobron, and F. Chmelik:

Phys. Rev. E, 2013, vol. 88, pp. 042402 (8).

44. H. Jiang Q. Zhang, X. Chen, Z. Chen, Z. Jiang, X. Wu, and J. Fan: Acta Mater., 2007, vol. 55, pp. 2219-28.

45. S. Fu, T. Cheng, Q. Zhang, Q. Hu, and P. Cao: Acta Mater., 2012, vol. 60, pp. 6650-56.

46. P. G. McCormick: Scripta metall., 1978, vol. 12, pp. 197-200.

47. C.J. Pérez, Á Corral, A. Díaz-Guilera, K. Christensen, and A. Arenas: Int. J. Mod. Phys. $B$, 1996, vol. 10, pp. 1111-51.

48. D.A. Zhemchuzhnikova, M.A. Lebyodkin, T.A. Lebedkina, and R.O. Kaibyshev: Mater. Sci.

Eng. A, 2015, vol. 639, pp. 37-41. 


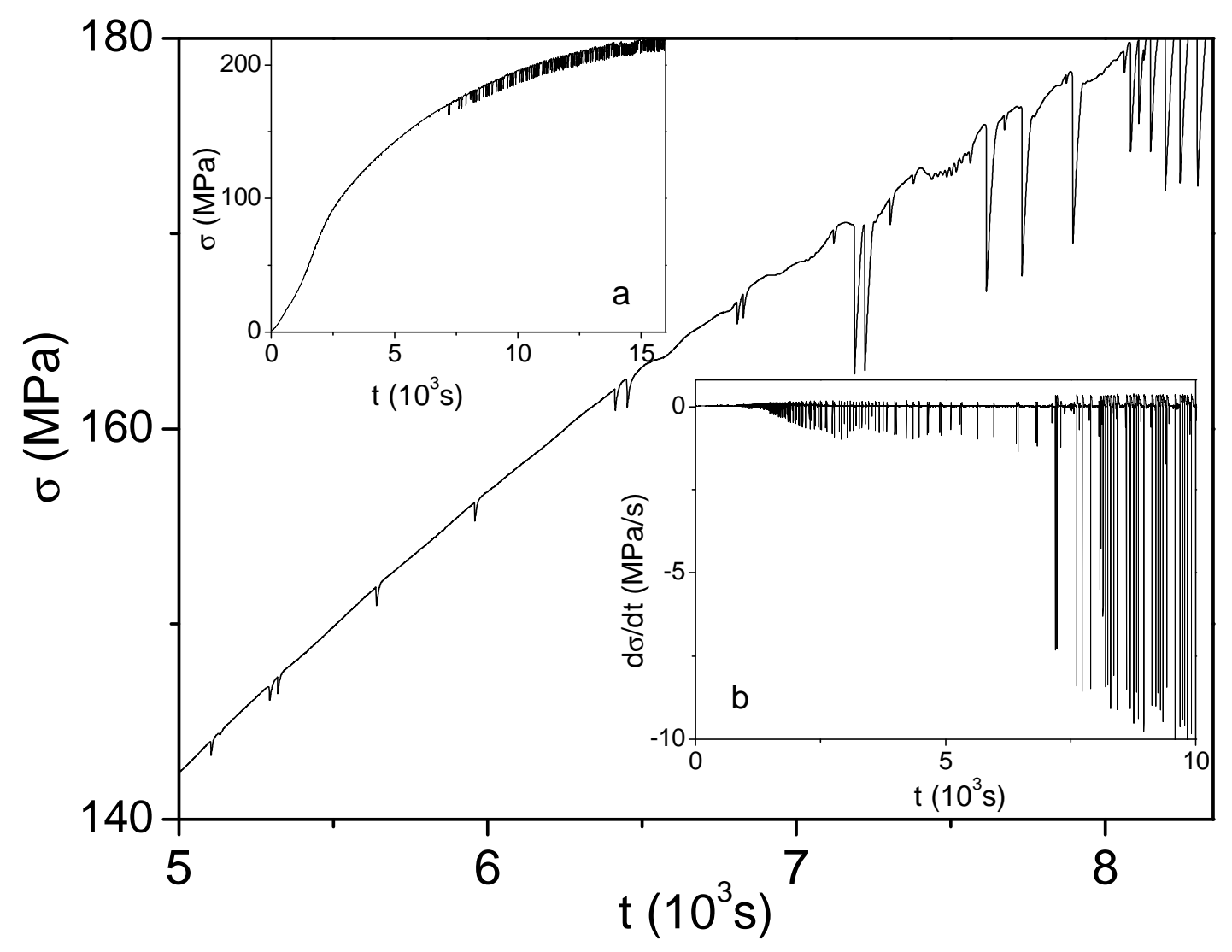

Figure 1. A portion of the deformation curve $\sigma(t)$ displaying small and large stress serrations. Inset: (a) the entire deformation curve; (b) time dependence of the derivative $d \sigma / d t$ during the initial portion of the deformation curve of an Al-3\% Mg sample $\left(\dot{\varepsilon}_{a}=2 \times 10^{-5} \mathrm{~s}^{-1}\right)$. The beginning of deep falls in $d \sigma / d t$ around $t \approx 6500 \mathrm{~s}$ corresponds to the conventional critical strain $\varepsilon_{c}$ for onset of type $C$ serrations. 


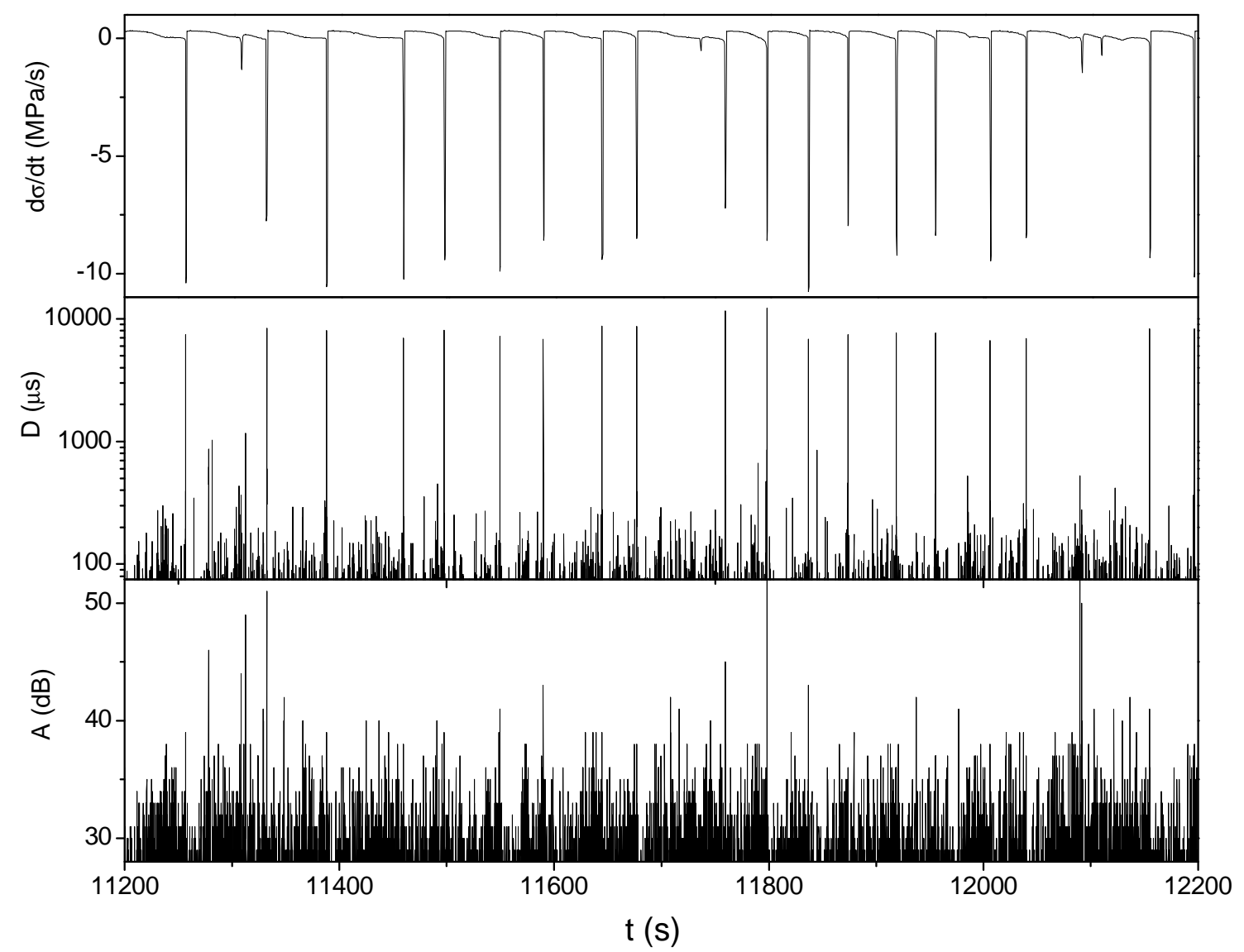

Figure 2. Time dependences of the time derivative $d \sigma / d t$ of the flow stress, duration $D$, and llogarithmic amplitude $A$ of AE events in an Al-3\% Mg specimen during the PLC effect $\left(\dot{\varepsilon}_{a}=2 \mathrm{x}\right.$ $\left.10^{-5} \mathrm{~s}^{-1}\right)$. 

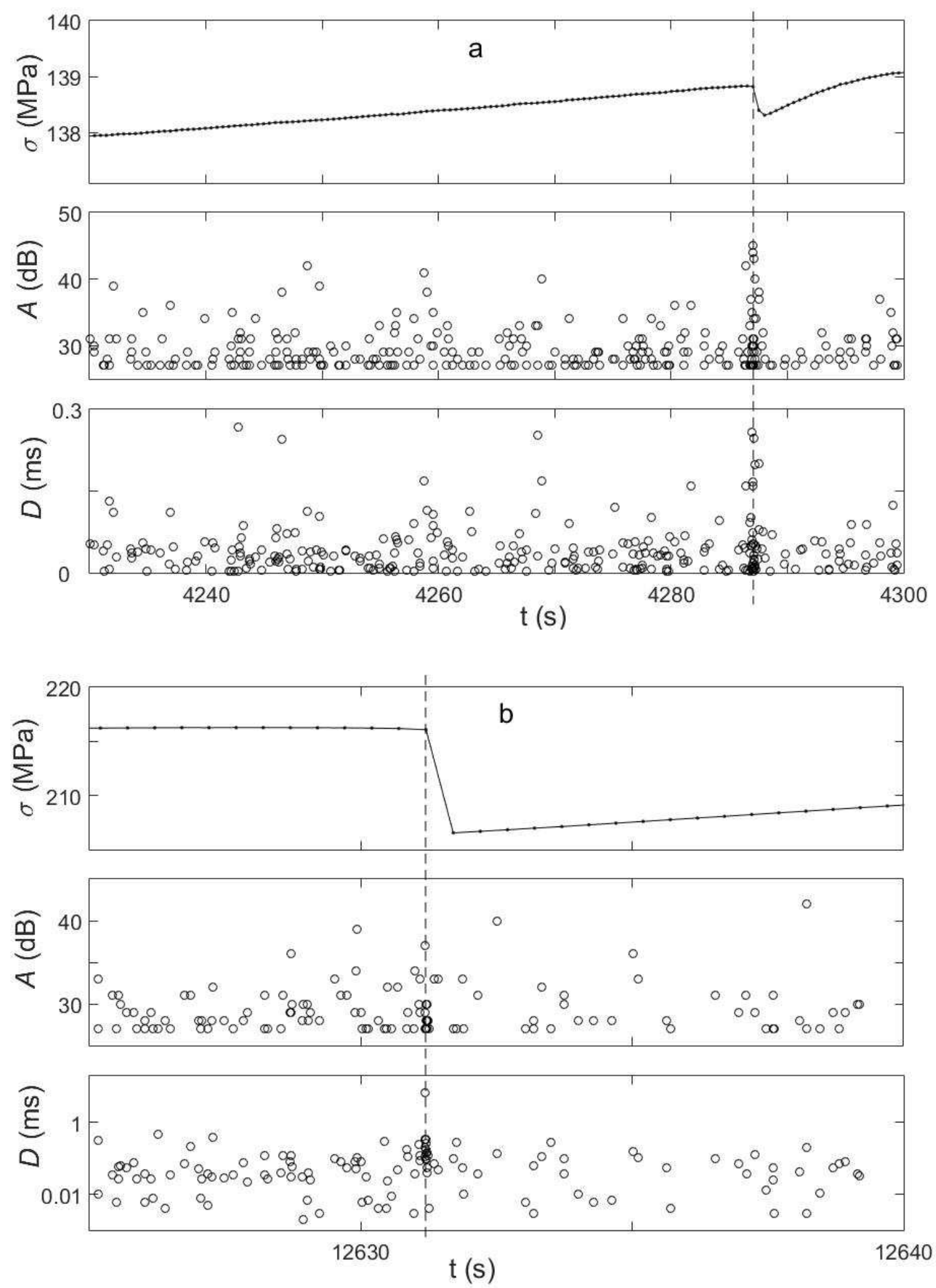

Figure 3. Portion of a deformation curve $\sigma(t)$, amplitude $A$, and duration $D$ of the accompanying AE events for strain intervals (a) before the onset of deep PLC serrations and (b) during deep serrations. Note the logarithmic $D$-scale in Fig. 3 b. $\dot{\varepsilon}_{a}=2 \times 10^{-5} \mathrm{~s}^{-1}$. 


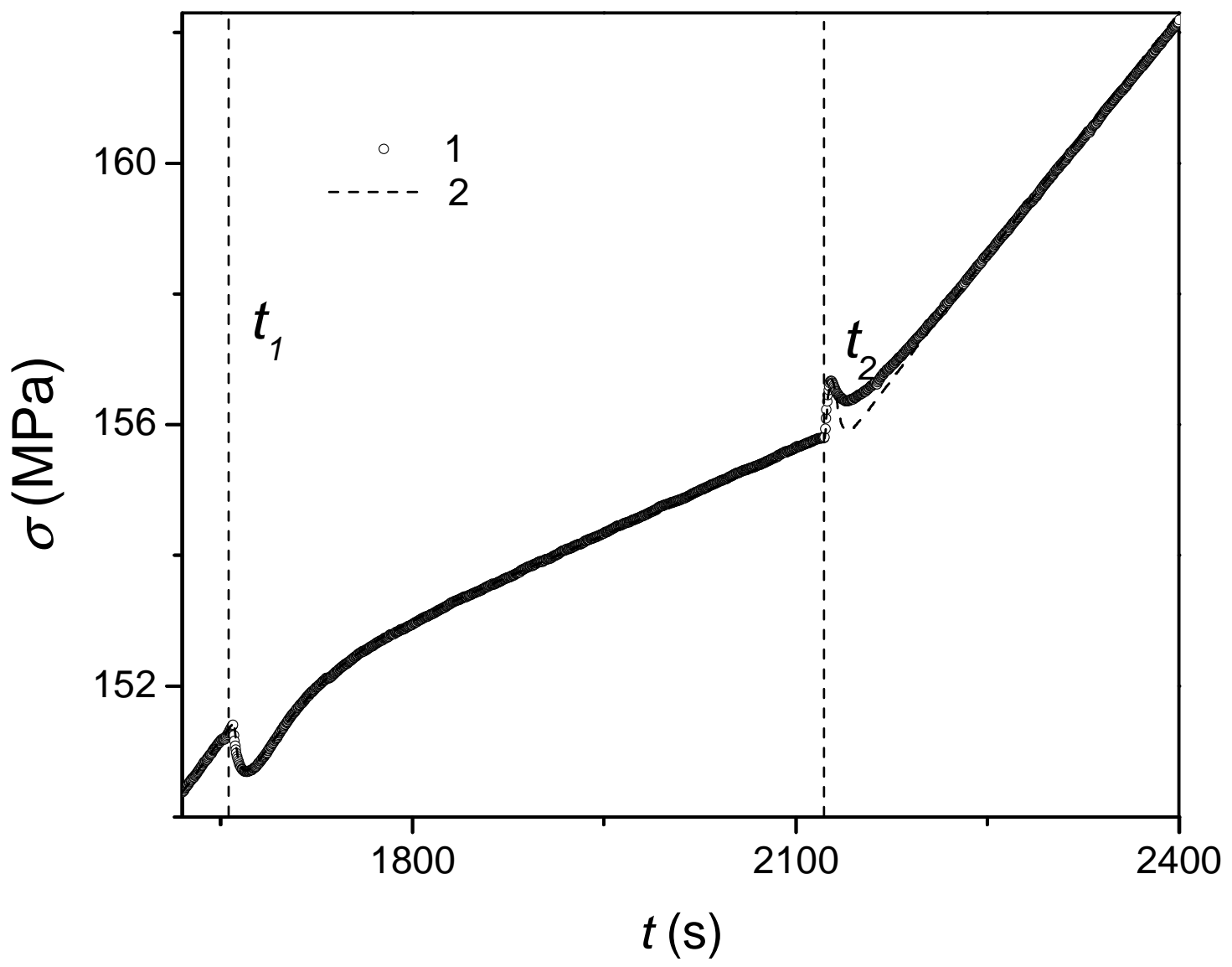

Figure 4. Time dependence of the flow stress after switching $\dot{\varepsilon}_{a}$ from $1.25 \times 10^{-5} \mathrm{~s}^{-1}$ to $4.15 \times 10^{-5} \mathrm{~s}^{-1}$ (at the instant $t_{1}$ ) and back (at the instant $t_{2}$ ). 1 - Experimental data, 2 - Results of calculation. 


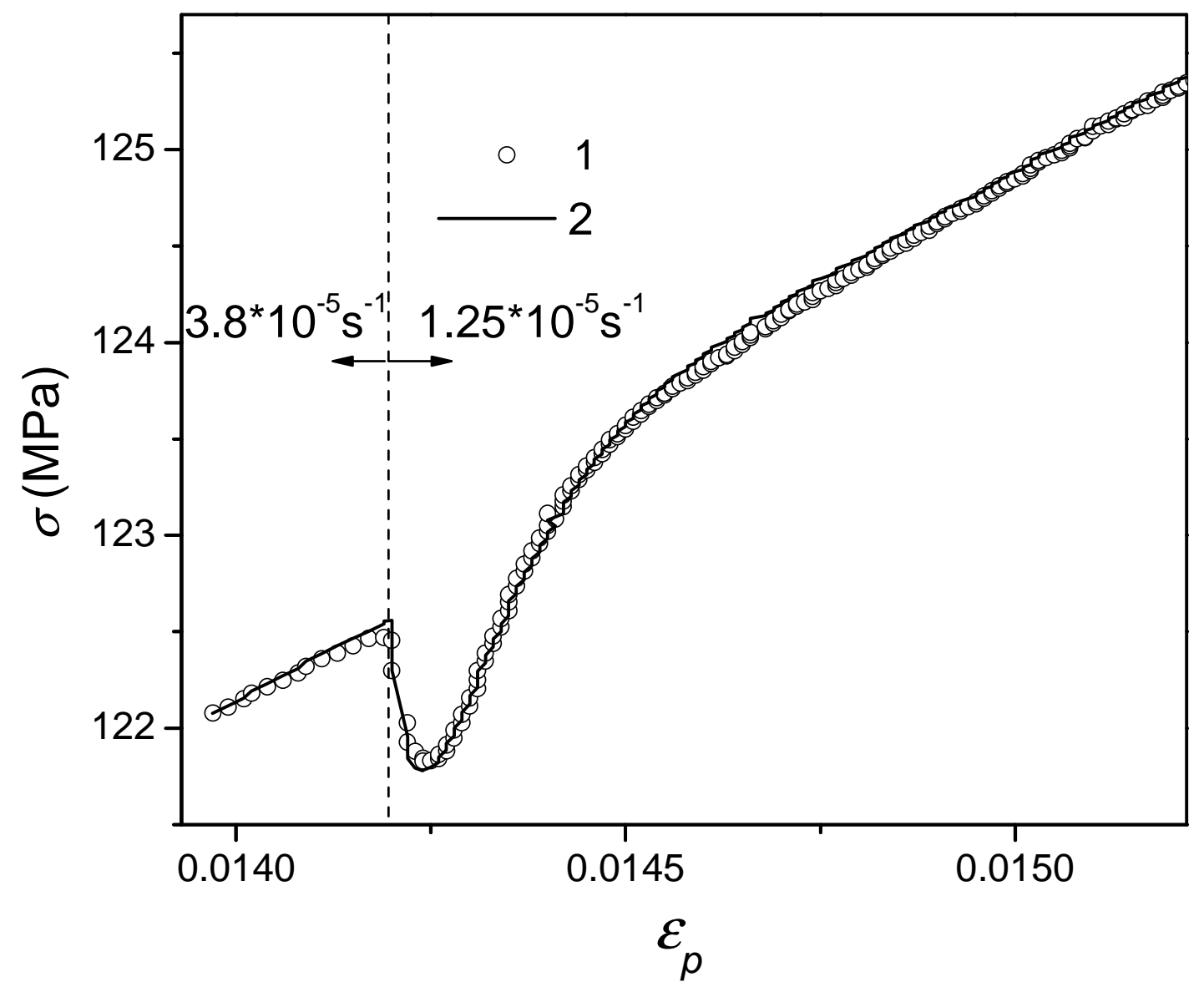

Figure 5. Dependence of the flow stress on $\varepsilon_{p}$ after switching of $\dot{\varepsilon}_{a}$-value. 1 - Experimental data, 2 - Results of calculation. 


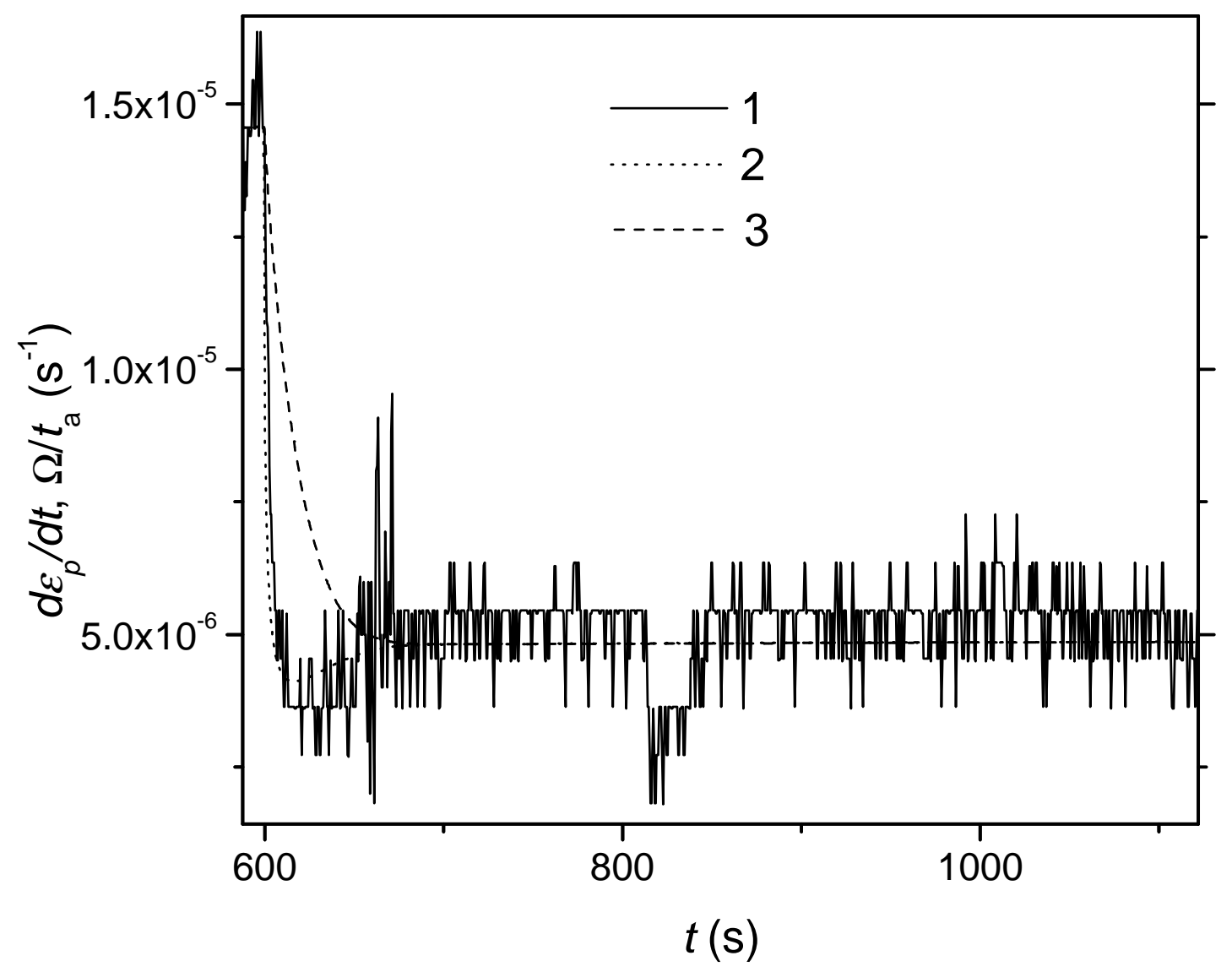

Figure 6. Evolution of the plastic strain rate after switching $\dot{\varepsilon}_{a}$ from $1.6 \times 10^{-5} \mathrm{~s}^{-1}$ to $5.3 \times 10^{-5} \mathrm{~s}^{-1} .1$ - Experimental data, 2 - Results of calculation, 3 - evolution of the ratio $\Omega / t_{a}$. 


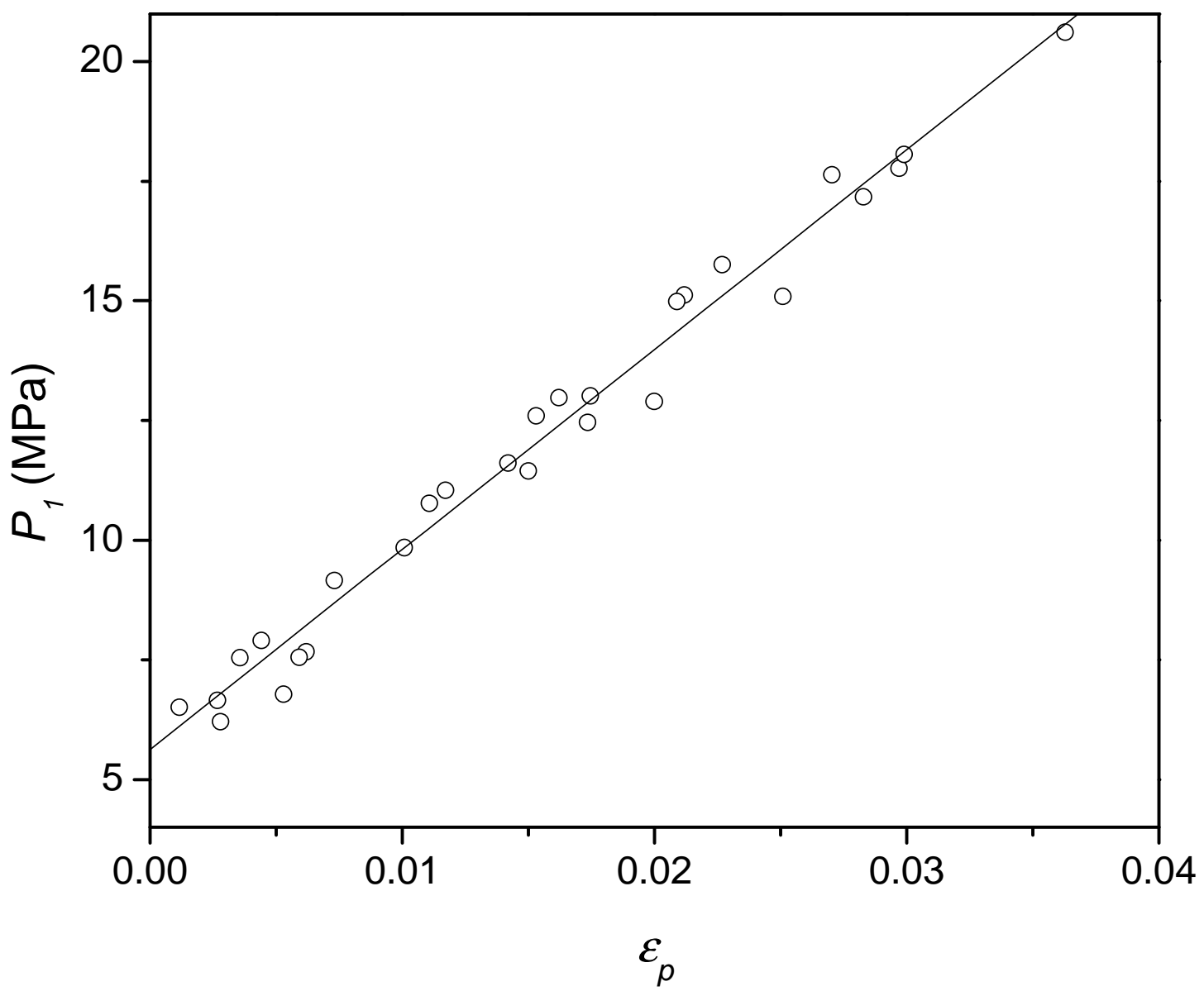

Figure 7. Dependence of the parameter $P_{1}$ on $\varepsilon_{p}$ (data for various samples and various $\dot{\varepsilon}_{a}$-values). 


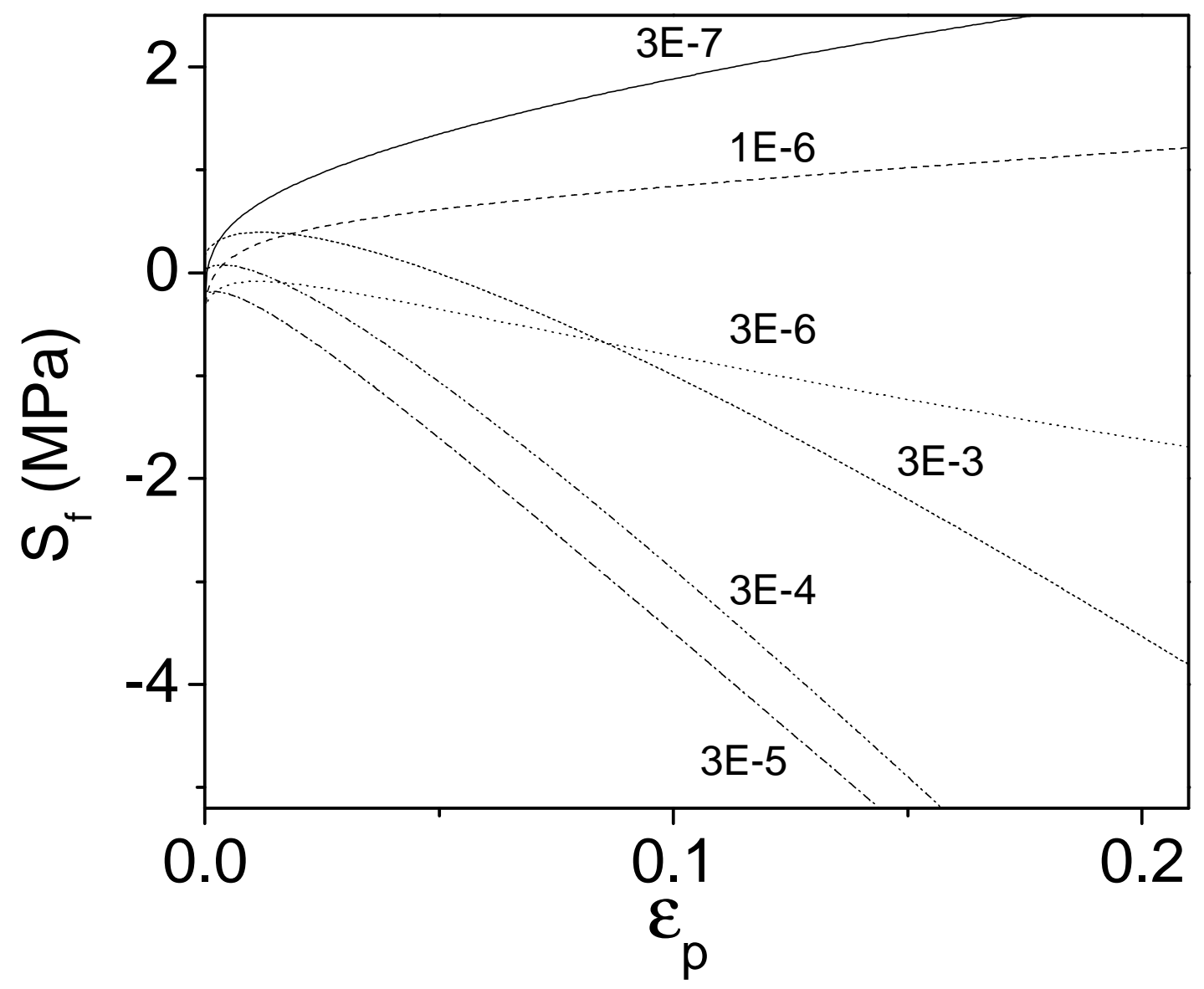

Figure 8. Strain dependences of the strain-rate sensitivity of the flow stress for various $\dot{\varepsilon}_{p}$ (numbers near the curves give $\dot{\varepsilon}_{p}$-values in $\mathrm{s}^{-1}$ ). 


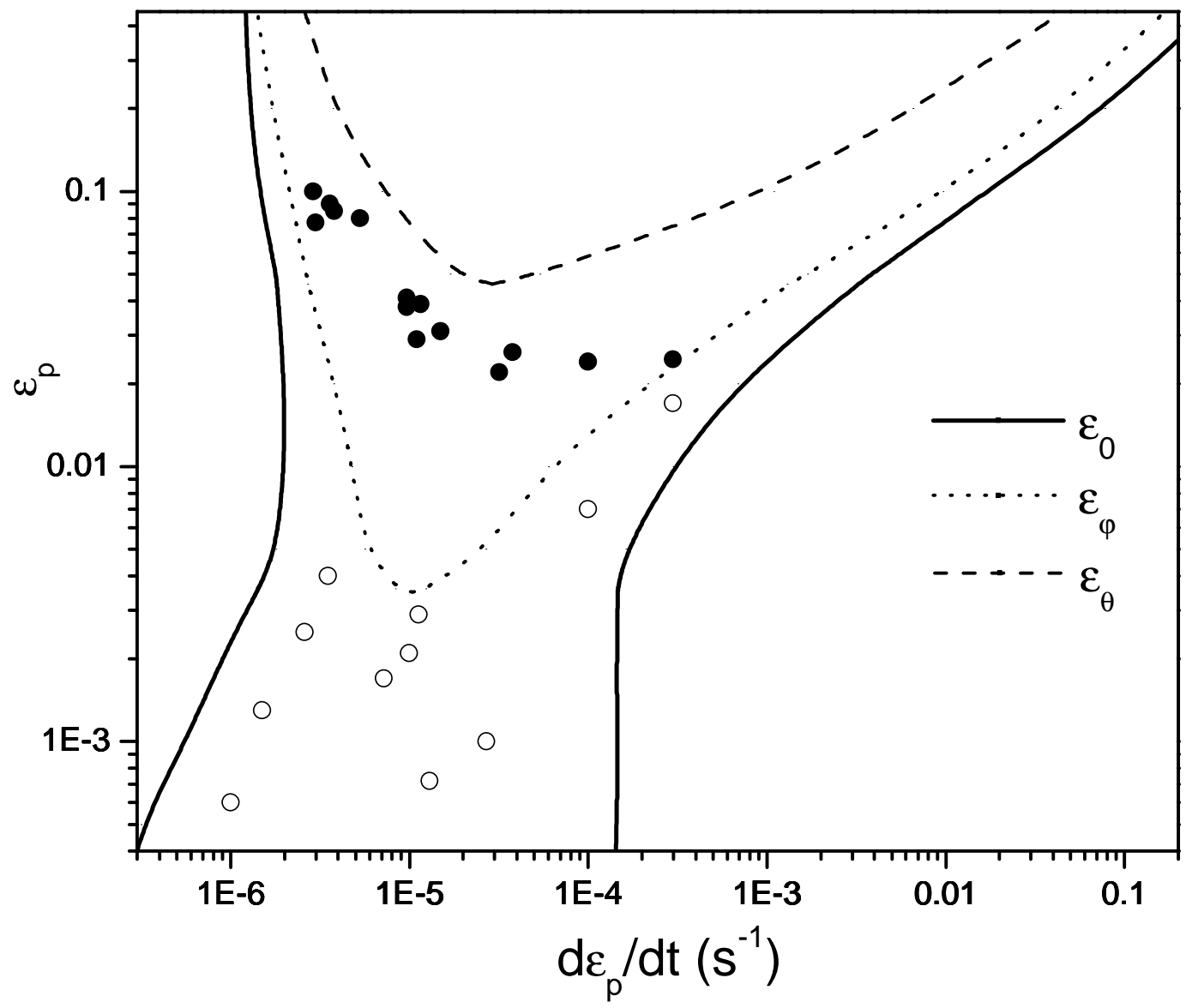

Figure 9. Experimental data for the critical strains $\varepsilon_{c}$ (solid symbols) and $\varepsilon_{S}$ (open symbols) corresponding, respectively, to onsets of deep and low-amplitude stress serrations. The data are plotted as functions of the plastic strain rate. The lines show the calculated boundaries of unstable plastic flow, corresponding to criteria (7) $\varepsilon_{0},(10) \varepsilon_{\phi}$, and (11) $\varepsilon_{\theta}$. In each case, unstable behavior corresponds to the regions inside the curves. 